\title{
Riqueza de fungos anamorfos na serapilheira de Manilkara maxima, Parinari alvimii e Harleyodendron unifoliolatum na Mata Atlântica do Sul da Bahia'
}

Dilze Maria Argôlo Magalhães ${ }^{2,3,4}$, Edna Dora Martins Newman Luz ${ }^{2,3}$, Albertí Ferreira Magalhães², Lindolfo Pereira dos Santos Filho² Leandro Lopes Loguercio ${ }^{3,}$ e José Luiz Bezerra ${ }^{2,3}$

Recebido em 16/03/2010. Aceito em 4/10/2011

\section{RESUMO}

(Riqueza de fungos anamorfos na serapilheira de Manilkara maxima, Parinari alvimii e Harleyodendron unifoliolatum na Mata Atlântica do Sul da Bahia). A maioria dos fungos tem a função de decompor moléculas orgânicas complexas possibilitando o aporte de nutrientes para o solo pelo processo de ciclagem e fertilização. Este estudo objetiva analisar a riqueza, freqüência, constância e a similaridade entre os fungos decompositores da serapilheira de três plantas endêmicas da Mata Atlântica do Sul da Bahia e em três áreas. Folhas em decomposição foram coletadas no período de dezembro/2007 a junho/2008, lavadas, colocadas em câmaras-úmidas e incubadas na temperatura ambiente. Obteve-se uma riqueza total de 52 espécies e 39 gêneros. A riqueza obtida nas áreas foi: Parque Estadual Serra do Conduru-PESC (78,8\%), Ecoparque (71,2\%) e Reserva Capitão (61,5\%). Em relação às plantas, Harleyodendron unifoliolatum Cowan apresentou uma riqueza de 75,0\%, Manilkara maxima Pennington $57,7 \%$ e Parinari alvimii Prance $42,3 \%$. Houve similaridade fúngica entre as plantas e entre as áreas. A maioria dos fungos apresentou frequência esporádica e constância acidental. Este estudo, pioneiro na Mata Atlântica do Sul da Bahia e em relação às plantas estudadas, contribui para o conhecimento dos fungos anamorfos existentes neste bioma, riquíssimo em biodiversidade.

Palavras-chave: biodiversidade, fungos decompositores, plantas endêmicas, riqueza, similaridade

\begin{abstract}
(Richness of anamorphic fungi on the litter of Manilkara maxima, Parinari alvimii and Harleyodendron unifoliolatum in the Atlantic Forest of southern Bahia). Most fungi have the function of breaking down complex organic molecules, enabling the supply of nutrients to the soil through the process of cycling and fertilization. This paper aimed to analyze the richness, frequency, constancy and similarity among decomposing fungi on the litter of three endemic trees in three areas of the Atlantic Forest in southern Bahia. Decaying leaves were collected from December 2007 to June 2008, washed, placed in wet chambers and incubated at room temperature. Fifty-two fungal species belonging to 39 genera were found. The richness of each area was the following: Parque Estadual Serra do Conduru (78,8\%), Ecoparque (71.2\%) and Reserva Capitão (61.5\%). In relation to plants, the richness of Harleyodendron unifoliolatum Cowan was $75.0 \%$, Manilkara maxima Pennington $57.7 \%$ and Parinari alvimii Prance $42.3 \%$. There was similarity between plants and between areas. The frequency of most fungi was sporadic. This pioneer work on these trees of the Atlantic Forest of southern Bahia contributes to the knowledge of the anamorphic fungi existing in this biologically rich biome.
\end{abstract}

Key words: biodiversity, decomposing fungi, endemic plants, richness, similarity

Parte da Dissertação de Mestrado do primeiro Autor

2 Comissão Executiva do Plano da Lavoura Cacaueira, Centro de Pesquisa do Cacau, Itabuna, BA, Brasil

3 Universidade Estadual Santa Cruz, Pós-Graduação em Genética e Biologia Molecular, Ilhéus, BA, Brasil

4 Autor para correspondência: dilze.argolo@yahoo.com.br 


\section{Introdução}

A biota da Mata Atlântica é extremamente diversificada. Com extensas áreas ainda pouco conhecidas do ponto de vista biológico, acredita-se que a região abrigue de 1 a $8 \%$ da biodiversidade mundial (Conservation International do Brasil 2003), possuindo a maior biodiversidade por hectare entre as florestas tropicais (Conti \& Furlan 2003).

O Sul da Bahia possui um conjunto de remanescente florestal de Mata Atlântica que abriga um dos mais importantes centros de biodiversidade e endemismo de todo o bioma. Nesta região, foram criadas reservas biologicamente prioritárias e viáveis para a conservação da biodiversidade da Mata Atlântica com o objetivo de manter ou restaurar a conectividade da paisagem e facilitar o fluxo genético entre populações, aumentando a chance de sobrevivência ao longo prazo das comunidades biológicas (Araujo et al. 1998). Dentre essas reservas criadas encontra-se o Parque Estadual Serra do Condurú, a Reserva Capitão e o Ecoparque de Una, que representam as florestas dessa região caracterizadas por árvores altas, com folhas sempre-verdes e abundância de epífitas. Conserva ainda uma grande diversidade de espécies de fauna e de flora como observado na Serra do Condurú, onde foram identificadas 456 espécies de árvores em apenas 01 hectare (Araujo et al. 1998), constituindo uma das maiores densidades de espécies arbóreas do mundo, sendo diversas delas endêmicas (Martini et al. 2007).

A análise da biodiversidade terá mais crédito quando maior número de grupos taxonômicos e funcionais de organismos heterotróficos for incluído nas investigações (Symonides 1992), uma vez que a escolha de locais para conservação da biodiversidade está baseada em classificação ou mapeamento vegetal, não levando em conta as comunidades microbianas. Entretanto observa-se que numa comunidade florestal existe uma interação intensa entre a vegetação, o solo, e organismos decompositores que se expressa no processo cíclico de entrada e saída de matéria do solo: a ciclagem de nutrientes minerais (Queiroz 1999). Neste contexto, a serapilheira depositada sobre o solo das florestas e os fungos decompositores que atuam neste substrato, tem papel fundamental na dinâmica desses ecossistemas (César 1993). Conhecendo as funções das espécies fúngicas nos ecossistemas, é possível avaliar devidamente qualquer tipo de impacto decorrente da intervenção antrópica. Deste modo, alterações podem ser evitadas ou minimizadas e as medidas da preservação ou recuperação dos ecossistemas tornam-se facilitadas (Milanez 1995). Os fungos desempenham papel fundamental na natureza, a decomposição, com capacidade de produção de enzimas o que permite que atuem em praticamente todos os substratos (Maia 2003).

No Brasil, estudos taxonômicos sobre fungos decompositores, embora com alguns trabalhos publicados, ainda são escassos, principalmente com relação aos fungos decompositores na Mata Atlântica. Estudos sobre fungos anamorfos em folhedo em regiões do estado da Bahia e na
Mata Atlântica têm contribuído para o conhecimento da diversidade de fungos existente nos ecossistemas brasileiros (Castañeda-Ruiz et al. 2001, 2003; Calduch et al. 2002; Gusmão et al. 2005, 2008; Cruz et al. 2007; Marques et al. 2008; Barbosa et al. 2008, 2009).

Portanto, por não haver estudo com os fungos anamorfos nessas reservas, objetivou-se estudar a riqueza, a freqüência, a constância e a similaridade dos fungos decompositores da serapilheira entre três espécies vegetais endêmicas: Manilkara maxima Pennington, Parinari alvimii Prance e Harleyodendron unifoliolatum Cowan, e entre as diferentes unidades de conservação da Mata Atlântica do Sul da Bahia.

\section{Material e métodos}

Três unidades de conservação do Bioma Mata Atlântica do Sul da Bahia foram selecionadas para as coletas da serapilheira: o Parque Estadual Serra do Conduru (PESC), localizado nos municípios de Uruçuca, Itacaré e Ilhéus (14'23' 07,7"S e 39०04'43,6”W), com 152 m de altitude, o Parque ecológico (Ecoparque) situado no município de Una (15 $10^{\prime} 49,2^{\prime \prime}$ e $\left.39^{\circ} 02^{\prime} 09,8^{\prime \prime} \mathrm{W}\right)$, com 50 m de altitude e a Reserva Capitão localizada no município de Itacaré (14 $21^{\circ}$ ' $16,8^{\prime \prime}$ e $\left.39^{\circ} 03^{\prime} 23,4^{\prime \prime} \mathrm{W}\right)$, com $132 \mathrm{~m}$ de altitude. São áreas de preservação ambiental mantendo grande diversidade biológica, porém pouco estudada, não havendo nenhum estudo sobre a diversidade de fungos anamorfos. O PESC é uma unidade de conservação de proteção integral, sob a responsabilidade da Secretaria Estadual do Meio Ambiente; as reservas privadas do patrimônio natural Ecoparque e Reserva Capitão estão sob a responsabilidade do Instituto de Estudos Socioambientais do Sul da Bahia (IESB).

Entre as espécies vegetais endêmicas da Mata Atlântica destacam-se Harleyodendron unifoliolatum Cowan, da família Leguminosae, Manilkara maxima Pennington (Massaranduba) da família Sapotaceae e, Parinari alvimii Prance (Oiti cumbuca) da família Chrysobalanaceae, cujas características são: plantas raras, de ampla distribuição geográfica, de grande porte e com os tipos de folhas facilmente identificáveis no interior da mata. Estas espécies são encontradas nas três unidades de conservação da Mata Atlântica do Sul da Bahia.

As coletas foram realizadas nos meses de dezembro/2007, fevereiro/abril e junho/2008. Neste período no PESC houve uma variação de temperatura de 21,7-24,8 ${ }^{\circ} \mathrm{C}$, a umidade variou de $82,6-88,4 \%$ e a precipitação pluviométrica de 76,1-192,3 mm. No Ecoparque houve neste mesmo período uma variação de temperatura 23,2-25,4 ${ }^{\circ} \mathrm{C}$, umidade de $83,2-84,3 \%$ e precipitação de $38,8-214,6$ $\mathrm{mm}$. Enquanto que na Reserva Capitão no mesmo período a temperatura variou de $23,5-25,5^{\circ} \mathrm{C}$, a umidade de $79,2-83,4 \%$ e a precipitação variou de $83-133,6 \mathrm{~mm}$. No total foram feitas quatro coletas, das três espécies vegetais e de três indivíduos de cada espécie, em cada uma das três 
unidades de conservação escolhidas. Na primeira coleta as plantas foram identificadas, localizadas com GPS e marcadas com uma fita plástica laranja. Na serapilheira destas árvores foi arremessado, de forma aleatória, um quadrado $50 \mathrm{~cm}$ x $50 \mathrm{~cm}$ e coletada ao acaso uma folha por vez, sendo um total de dez folhas (uma amostra) em diferentes estágios de decomposição, totalizando 108 amostras.

As folhas em decomposição coletadas foram guardadas em sacos de papel (tipo Kraft), transportadas ao Laboratório de Biodiversidade de Fungos do Cepec-Ceplac, colocadas em vasilhames plásticos previamente perfurados onde foram lavadas por uma hora em água corrente, acondicionadas em câmaras-úmidas e incubadas à temperatura ambiente, as quais foram abertas diariamente por quinze minutos, para troca de ar (Castañeda-Ruiz et al. 2006). Após $72 \mathrm{~h}$ o material incubado foi observado em estereomicroscópio e revisado diariamente, durante 30 dias. Os microfungos foram retirados com o auxilio de uma agulha fina e colocados em lâminas com meio de montagem semi-permanente (resina PVL: álcool polivinílico + lactofenol) (Trappe \& Schenck 1982). Os fungos foram estudados em microscópio óptico e identificados utilizando bibliografia específica. Após a identificação, foram inoculados em meio de cultura batatadextrose-agar para o crescimento do micélio. As folhas colonizadas foram posteriormente desidratadas e convertidas em exsicatas sendo depositadas na coleção micológica do herbário André Maurício Vieira de Carvalho do Centro de Pesquisa de Cacau (Cepec).

Os aspectos ecológicos relativos à Riqueza, Frequência relativa, Constancia e Similaridade, foram calculados, segundo as fórmulas: Riqueza é definida como o número total de espécies encontradas em cada espécie vegetal e em cada área. Frequência relativa é calculada baseada na fórmula $\mathrm{F}=\mathrm{n} \times 100 / \mathrm{N}$, onde $\mathrm{n}$ significa o número de amostras em que uma espécie foi encontrada e $\mathrm{N}=$ total de amostras em cada espécie vegetal (3 espécimes x 3 áreas $x 4$ coletas $=36$ amostras) ou em cada área (3 espécies vegetais $\mathrm{x} 3$ espécimes de cada $\mathrm{x} 4$ coletas $=36$ amostras). Foram determinadas as seguintes classes de frequência: Esporádica, $\mathrm{F} \leq 10 \%$; Pouco frequente, $10<$ $\mathrm{F} \leq 30 \%$; Frequente, $30<\mathrm{F} \leq 70 \%$ e Muito frequente $\mathrm{F}>$ $70 \%$ (Dajoz 1983). Constância é calculada utilizando a fórmula $\mathrm{C}=\mathrm{p} \cdot 100 / \mathrm{P}$ onde, $\mathrm{p}=$ número de coletas em que uma espécie fúngica foi encontrada e $\mathrm{P}=$ número total de coletas. A constância foi classificada como: Acidental, C $\leq 25 \%$; Acessória, $25<\mathrm{C} \leq 50 \%$, Constante, C > 50\% (Cavalcanti \& Mobim 2004). A similaridade entre as áreas foi obtida através do Índice de Sorensen (Muller-Dombois, 1981 ), onde $S=2 c .100 / a+b$ onde, $c=$ número de fungos comuns a duas espécies vegetais ou a duas áreas e $\mathrm{a}+\mathrm{b}=$ número de fungos presentes nas plantas ou nas áreas; para as três espécies vegetais estudadas ou as três áreas usa-se a fórmula $\mathrm{S}=3 \mathrm{~d} \cdot 100 / \mathrm{a}+\mathrm{b}+\mathrm{c}$ onde, $\mathrm{d}=$ número de fungos comuns a três espécies vegetais ou a três áreas e $\mathrm{a}+\mathrm{b}+\mathrm{c}=$ número de fungos presentes nas plantas ou nas áreas

\section{Resultados e discussão}

No presente estudo foram obtidos 52 táxons, pertencentes a 39 gêneros de fungos anamorfos (Tab. 1). A riqueza encontrada neste estudo é semelhante a alguns resultados obtidos na Mata Atlântica como o de Marques et al. (2008) que observando folhas, pecíolos, galhos e cascas em dois fragmentos de Mata Atlântica, na Serra da Jibóia, Bahia, obtiveram como riqueza 106 espécies de fungos. Enquanto que Barbosa (2007), analisando folhas em decomposição de Clusia melchiori e C. nemorosa, também na Serra da Jibóia encontrou 79 táxons e Silva (2007), analisando o folhedo de Caesalpinia echinata, em duas áreas com e sem impacto de poluição aérea em São Paulo, obteve uma riqueza de 33 espécies.

Dos 52 táxons (Tab. 1), 39 foram encontrados associados à serapilheira de Harleyodendron unifoliolatum o que corresponde a uma riqueza de $75,0 \%$. Na serapilheira de Manilkara. maxima a riqueza foi de $57,7 \%$ com 30 táxons observados, enquanto que na serapilheira de Parinari alvimii foi obtida menor riqueza, $42,3 \%$ com a presença de 22 táxons (Tab. 2). Calculando-se a riqueza nas áreas de estudo, no PESC obteve-se 78,6\% (41 táxons), no Ecoparque 71,2\% (37 táxons) e na Reserva Capitão 61,5\% da riqueza total, com 32 táxons (Tab. 2).

Analisando a riqueza das espécies vegetais por local (Fig. 1) foi observado que no PESC, Harleyodendron unifoliolatum apresentou a maior riqueza $(59,6 \%)$ comparada às outras espécies vegetais, Manilkara maxima $(38,5 \%)$ e Parinari alvimii (25\%). Harleyodendron unifoliolatum apresentou maior riqueza no PESC comparada às outras áreas, Ecoparque (34,6\%) e Reserva Capitão (28,8\%). Manilkara maxima apresentou riqueza bem próxima em termos percentuais quando analisada nas três áreas (PESC $=38,5 \%$; Ecoparque $=42,3 \%$; Reserva Capitão $=30,8 \%$ ), diferente dos resultados obtidos para Parinari alvimii e Harleyodendron unifoliolatum (Fig. 1). Parinari alvimii apresentou menor riqueza no PESC $(25,0 \%)$ e na Reserva Capitão $(15,4 \%)$, enquanto no Ecoparque a riqueza apresentou-se idêntica àquela obtida por Harleyodendron unifoliolatum (34,6\%).

Em termos percentuais, obteve-se a mesma riqueza nas três áreas (Tab. 2), praticamente a mesma quantidade de espécies, porém com diferenças na sua composição. Resultado similar foi obtido por Marques et al. (2008) estudando uma área de mata fechada $(\mathrm{A})$ e outra de mata com árvores esparsas (B) de Mata Atlântica na Bahia, onde foi encontrada na área $\mathrm{A}$ uma riqueza de 75 táxons e na Área B 69 táxons. Outros trabalhos também mostram maior ou menor grau de riqueza. Contudo, quando se observa a importância da biodiversidade para a conservação do ecossistema, verificase que quanto maior o número de espécies por unidade de área maior a probabilidade de assegurar uma elevada representação de caracteres genéticos, visto que os ecossistemas são tanto mais estáveis, quanto maior a diversidade no sistema (Scott et al. 1987; Myers 1988, 1990). 
Tabela 1. Fungos anamorfos identificados na serrapilheira de Harleyodendron unifoliolatum Cowan (I) Manilkara maxima Penn. (II) e Parinari alvimii Prance (III), nas três áreas da Mata Atlântica do Sul da Bahia: PESC, Ecoparque e Reserva Capitão.

\begin{tabular}{|c|c|c|c|c|c|c|c|c|c|}
\hline \multirow{2}{*}{ Táxons } & \multicolumn{3}{|c|}{ PESC/plantas } & \multicolumn{3}{|c|}{ Ecoparque/plantas } & \multicolumn{3}{|c|}{ R. Capitão/plantas } \\
\hline & $\mathrm{I}$ & II & III & I & II & III & $\mathrm{I}$ & II & III \\
\hline Anungitea sp. & $\mathrm{X}$ & & & & & & $\mathrm{X}$ & & \\
\hline Ascochyta sp. & & & & $\mathrm{X}$ & & & & & \\
\hline Atrosetaphiale flagelliformis (Matsush.) Matsush. & $\mathrm{X}$ & $\mathrm{X}$ & & $\mathrm{X}$ & $\mathrm{X}$ & $\mathrm{x}$ & & $\mathrm{X}$ & \\
\hline Beltrania querna Harkn. & & & & & & & & & $\mathrm{X}$ \\
\hline Beltrania rhombica Penz. & $\mathrm{X}$ & $\mathrm{X}$ & & $\mathrm{X}$ & $\mathrm{X}$ & & $\mathrm{X}$ & $\mathrm{X}$ & \\
\hline Beltraniella fertilis Heredia, R.M. Arias, M. Reyes \& R.F. Castañeda & & & & & & $\mathrm{x}$ & & & \\
\hline Beltraniella portoricensis (F.Stevens) Piroz. \& S.D. Patil & $\mathrm{X}$ & $\mathrm{X}$ & & $\mathrm{X}$ & $\mathrm{X}$ & & $\mathrm{X}$ & $\mathrm{X}$ & \\
\hline Chaetosphaeria innumera (Berk. \& Broome) Tul. \& C. Tul. & $\mathrm{X}$ & & & & & & & & \\
\hline Circinotrichum falcatisporum Piroz. & $\mathrm{X}$ & & & $\mathrm{X}$ & $\mathrm{X}$ & & $\mathrm{x}$ & & \\
\hline Cladosporium tenuissimum Cooke & & & $\mathrm{X}$ & & & & & $\mathrm{X}$ & \\
\hline Cryptophiale kakombensis Piroz. & $\mathrm{X}$ & $\mathrm{X}$ & & & $\mathrm{X}$ & & & $\mathrm{X}$ & \\
\hline Cryptophiale udagawae (Piroz. \& Ichinoe) Piroz. & $\mathrm{X}$ & $\mathrm{X}$ & & & $\mathrm{X}$ & & & $\mathrm{X}$ & \\
\hline Dactylaria pseudomanifesta D.M.A. Magalhães \& J.L. Bezerra & $\mathrm{X}$ & & & & $\mathrm{X}$ & & & & \\
\hline Dictyosporium elegans Corda & & $\mathrm{X}$ & & & & & & $\mathrm{X}$ & \\
\hline Dictyosporium oblongum (Fuckel) S. Hughes & $\mathrm{X}$ & & & & & & & & \\
\hline Elachopeltis sp. & $\mathrm{X}$ & & & & & & $\mathrm{X}$ & & \\
\hline Geotrichum sp. & $\mathrm{X}$ & $\mathrm{X}$ & & $\mathrm{X}$ & $\mathrm{X}$ & & & $\mathrm{X}$ & \\
\hline Gliocladium roseum Bainier & & & & $\mathrm{X}$ & & & $\mathrm{X}$ & & \\
\hline Grallomyces portoricensis F. Stevens & $\mathrm{X}$ & $\mathrm{X}$ & $\mathrm{X}$ & & $\mathrm{X}$ & $\mathrm{x}$ & & & $\mathrm{X}$ \\
\hline Gyrothryx grisea Piroz. & $\mathrm{X}$ & & & $\mathrm{X}$ & $\mathrm{X}$ & & $\mathrm{X}$ & & \\
\hline Gyrothrix magica (Lunghini \& Onofri) Onofri \& Lunghini & $\mathrm{X}$ & $\mathrm{X}$ & $\mathrm{X}$ & $\mathrm{X}$ & $\mathrm{X}$ & $\mathrm{x}$ & & $\mathrm{X}$ & \\
\hline Gyrothrix verticiclada (Goid.) S. Hughes \& Piroz & $\mathrm{X}$ & & & & & & & & \\
\hline Helicosporium guianensis Linder & $\mathrm{X}$ & $\mathrm{X}$ & $\mathrm{X}$ & & $\mathrm{X}$ & & & $\mathrm{X}$ & $\mathrm{X}$ \\
\hline Helicosporium sp. & $\mathrm{X}$ & & & $\mathrm{X}$ & & & & & \\
\hline Humicola sp. & & & & & $\mathrm{X}$ & & & & \\
\hline Idriella acerosa R.F. Castañeda \& W.B. Kendr. & $\mathrm{X}$ & $\mathrm{X}$ & $\mathrm{X}$ & & & $\mathrm{X}$ & & $\mathrm{X}$ & \\
\hline Idriella sp. & $\mathrm{X}$ & & $\mathrm{X}$ & & $\mathrm{X}$ & $\mathrm{x}$ & $\mathrm{X}$ & & \\
\hline Isthmotricladia sp. & & $\mathrm{X}$ & & & & $\mathrm{X}$ & & & \\
\hline Inesiosporium longispirale (R.F. Castañeda) R.F. Castañeda & $\mathrm{X}$ & $\mathrm{X}$ & $\mathrm{X}$ & & $\mathrm{X}$ & $\mathrm{X}$ & & $\mathrm{X}$ & \\
\hline Lasiodiplodia sp. & & & & $\mathrm{x}$ & & & & & \\
\hline Mariannaea sp. & & & & $\mathrm{x}$ & & & $\mathrm{X}$ & & \\
\hline Paliphora intermedia Alcorn & & $\mathrm{X}$ & & & & & & & \\
\hline Paraceratocladium polysetosum R.F. Castañeda & $\mathrm{X}$ & $\mathrm{X}$ & $\mathrm{X}$ & $\mathrm{x}$ & $\mathrm{X}$ & $\mathrm{x}$ & & & $\mathrm{x}$ \\
\hline Paraceratocladium silvestre R.F. Castañeda & & $\mathrm{X}$ & & & $\mathrm{X}$ & & & $\mathrm{X}$ & \\
\hline Pestalotiopsis sp. & & & & & & $\mathrm{x}$ & $\mathrm{X}$ & $\mathrm{X}$ & $\mathrm{x}$ \\
\hline Pseudobeltrania macrospora Heredia, R.M. Arias, M. Reyes, R.F. Castañeda & & & & & & & & & $\mathrm{X}$ \\
\hline Pseudobotrytis terrestris (Timonin) Subram. & $\mathrm{X}$ & & & & & & & & \\
\hline Pseudodictyosporium wauense Matsush. & & & & $\mathrm{x}$ & & & & & \\
\hline Scolecobasidium aff dendroides Piroz. \& Hodges & $\mathrm{X}$ & & & & & & $\mathrm{X}$ & & \\
\hline Scolecobasidium cf. tropicum Matsush. & & & & & $\mathrm{X}$ & & & & \\
\hline Speiropsis scopiformis Kuthub. \& Nawawi & $\mathrm{X}$ & & & & & & $\mathrm{X}$ & & \\
\hline Sporidesmiella aspera Kuthub. \& Nawawi & $\mathrm{X}$ & $\mathrm{X}$ & & & & $\mathrm{x}$ & & & \\
\hline Sporidesmiella vignalensis W.B. Kendr. \& R.F. Castañeda & $\mathrm{X}$ & & $\mathrm{X}$ & & $\mathrm{X}$ & $\mathrm{x}$ & & & $\mathrm{x}$ \\
\hline Sporidesmium bambusae M.B. Ellis & & & $\mathrm{X}$ & & & $\mathrm{X}$ & & & \\
\hline Sporidesmium coffeicola M.B. Ellis & $\mathrm{X}$ & & & & & & & & \\
\hline Trichoderma sp. & $\mathrm{X}$ & & $\mathrm{X}$ & $\mathrm{x}$ & & $\mathrm{x}$ & $\mathrm{X}$ & & \\
\hline Triscelophorus curviramifer (Matsush.) Matsush. & & $\mathrm{X}$ & & & & $\mathrm{X}$ & & & \\
\hline Triscelophorus deficiens (Matsush.) Matsush. & & $\mathrm{X}$ & & & & $\mathrm{x}$ & & & \\
\hline Vermiculariopsiella cornuta (V.Rao \& de Hoog) Nawawi,Kuthub. \& B. Sutton & $\mathrm{X}$ & & & & & & & & \\
\hline Volutella minima Höhn & & & & $\mathrm{x}$ & $\mathrm{X}$ & & $\mathrm{X}$ & & \\
\hline Volutella sp. & $\mathrm{X}$ & $\mathrm{X}$ & $\mathrm{X}$ & $\mathrm{x}$ & $\mathrm{X}$ & $\mathrm{x}$ & $\mathrm{X}$ & $\mathrm{X}$ & $\mathrm{X}$ \\
\hline Zygosporium gibbum (Sacc., M. Rousseau \& E. Bommer) S. Hughes & & & $X$ & $\mathrm{X}$ & $\mathrm{X}$ & $\mathrm{X}$ & & $\mathrm{X}$ & \\
\hline Total & 31 & 20 & 13 & 18 & 22 & 18 & 15 & 16 & 8 \\
\hline
\end{tabular}


Tabela 2. Riqueza fúngica obtida na serrapilheira de Harleyodendron unifoliolatum Cowan, Manilkara maxima Penn. e Parinari alvimii Prance, e nas três áreas da Mata Atlântica do Sul da Bahia: PESC, Ecoparque e Reserva Capitão

\begin{tabular}{lcccccccc}
\hline Local & $\begin{array}{c}\text { Harleyodendron } \\
\text { unifoliolatum }\end{array}$ & $(\%)$ & Manilkaramaxima & (\%) & Parinarialvimii & (\%) & Riqueza/área & (\%) \\
\hline PESC & 31 & 59,6 & 20 & 38,5 & 13 & 25,0 & 41 \\
Ecoparque & 18 & 34,6 & 22 & 42,3 & 18 & 34,6 & 37 \\
R. Capitão & 15 & 28,8 & 16 & 30,8 & 8 & 15,4 & 32 & 71,2 \\
Riqueza / planta & 39 & 75,0 & 30 & 57,7 & 22 & 42,3 & 52 & $100 \%$ \\
\hline
\end{tabular}

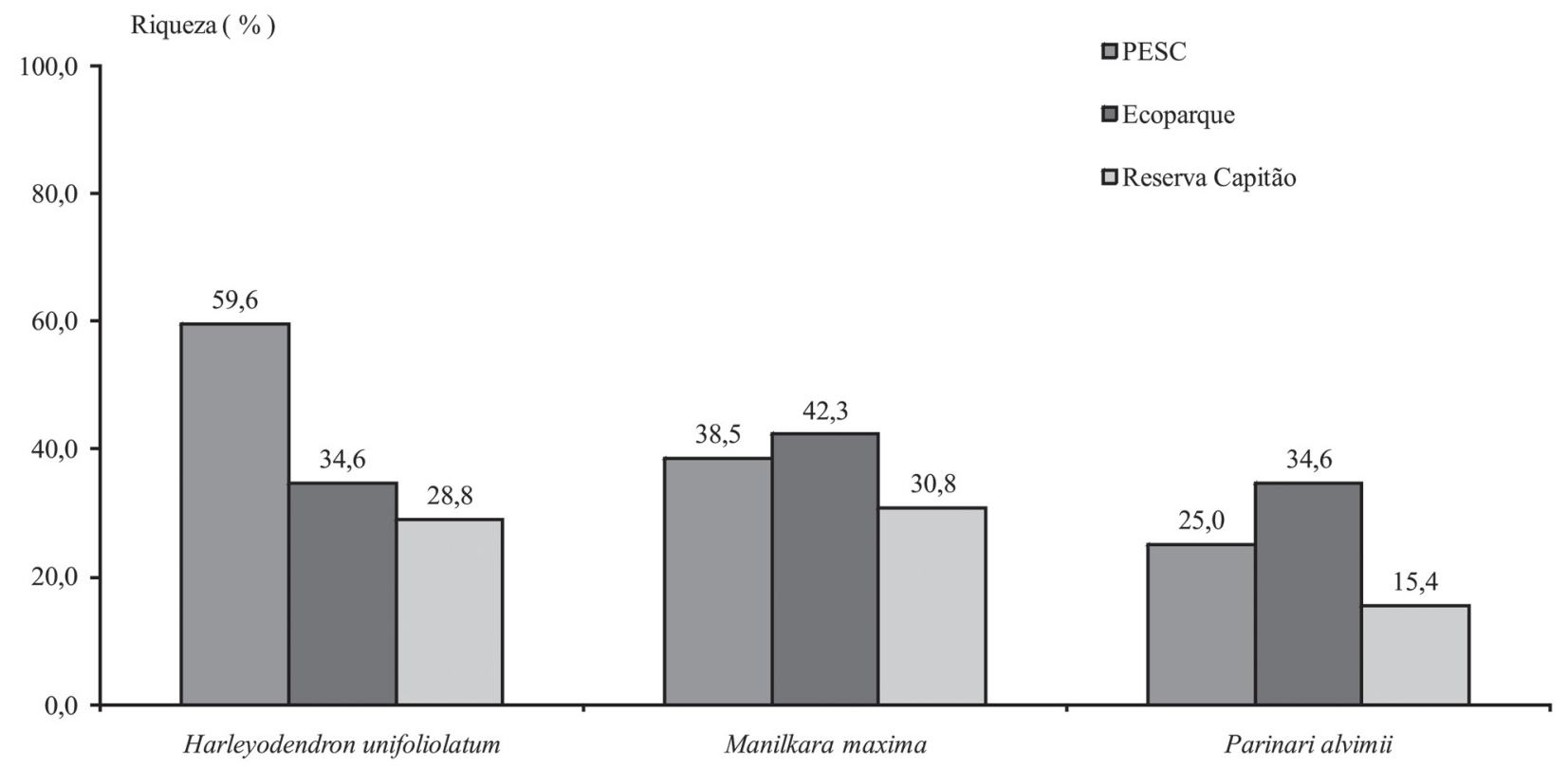

Espécies vegetais

Figura 1. Riqueza fúngica encontrada na serapilheira de Harleyodendron unifoliolatum Cowan, Manilkara maxima Penn. e Parinari alvimii Prance, no PESC, Ecoparque e Reserva Capitão da Mata Atlântica do Sul da Bahia

Quando comparada a riqueza entre as plantas o mesmo resultado não foi observado, visto que as três espécies vegetais pertencem a gêneros e famílias diferentes. Harleyodendron unifoliolatum apresentou a maior riqueza analisando as três áreas, comparando com a riqueza geral de Manilkara máxima e Parinari alvimii. Mas quando analisada a riqueza por área, esta espécie apresentou a maior riqueza somente no PESC; no Ecoparque e na Reserva Capitão Manilkara maxima apresentou maior riqueza comparada às duas outras espécies vegetais. Também foi observado que na maioria das coletas a serapilheira de Manilkara maxima e de Parinari alvimii apresentavam sempre maior quantidade de folhas recém caídas, enquanto que Harleyodendron unifoliolatum além de ter folhas mais finas e de tamanho maior na serapilheira as folhas caídas eram escassas e apresentavam um estado de decomposição mais avançado. Nas serapilheiras das outras duas espécies a abundância de folhas pode levar a necessidade provável de maior tempo tanto para a colonização pelos fungos como para a decomposição. Além disto, Parinari alvimii conhecida vulgarmente por oiti cumbuca por causa do formato em cumbuca das folhas, $o$ que, provavelmente, dificulta contato total da folha com o solo, necessitando de tempo maior para a decomposição.

As folhas de Manilkara maxima e Harleyodendron unifoliolatum preservavam umidade por mais tempo, enquanto as de Parinari alvimii eram secas, mesmo quando conservadas em câmara-úmida, e apresentavam pêlos na parte abaxial das folhas dificultando a visualização e retirada dos fungos. Este fato pode também ser uma justificativa para a maior riqueza de fungos na serapilheira de Harleyodendron unifoliolatum e Manilkara maxima e menor em Parinari alvimii. Segundo Dix \& Webster (1995) a estrutura e o tempo de senescência das folhas são fatores que podem contribuir para o crescimento dos fungos na serapilheira.

Dentre os 52 fungos obtidos, 25 táxons foram exclusivos e assim distribuídos: 16 em Harleyodendron unifoliolatum, 5 em Manilkara maxima e 4 espécies em Parinari alvimi (Tab. 1). A similaridade de fungos entre as espécies Harleyodendron unifoliolatum e Manilkara maxima foi de (60,9\%), entre Harleyodendron unifoliolatum e Parinari alvimii foi $45,9 \%$ e entre Manilkara maxima e Parinari alvimi de 61,5 $\%$. Comparando as três espécies vegetais obteve-se uma 
similaridade de 39,6\% (Fig. 2). A diferença nos valores de similaridades obtidos deve-se ao fato da riqueza também ter sido diferenciada entre essas espécies e isto provavelmente ocorre por serem espécies vegetais pertencentes a diferentes gêneros (Fig. 2). Maia (1983), pesquisando populações de fungos em folhas de Licania octandra (Hoffmgg. ex R. \& S.) Kuntze e L. kunthiana Hook encontrou similaridade de $(70,0 \%)$ entre elas. Mas quando estas espécies foram comparadas com a população de Hortia arborea Engl. a similaridade foi de $64,0 \%$ e $63,5 \%$, respectivamente.

Harleyodendron unifoliolatum apresentou mais espécies fúngicas exclusivas quando comparada a Manilkara maxima e Parinari alvimii (Tab. 1). Isto pode indicar uma preferência de algumas espécies de fungos por esta planta. Esta observação pode ser confirmada por Polishok et al. (1996), quando argumentam que existe a preferência dos fungos por plantas em particular, o que assegura a riqueza fúngica em folhedos mistos nos ecossistemas florestais.

A similaridade de fungos obtida entre as áreas foi elevada em todas as análises (Fig 2). Entre o PESC e Ecoparque (81,7\%), entre o PESC e Reserva Capitão (81,2\%), e entre o Ecoparque e Reserva Capitão (75,0\%) e a similaridade entre as três áreas foi de $67,6 \%$. Esta similaridade entre as áreas de estudo significa que mais de $50,0 \%$ das espécies foram encontradas nas três áreas. Contudo, Silva (2007), analisando o folhedo de Caesalpinia echinata, em duas áreas com e sem impacto de poluição aérea em São Paulo obteve um índice de similaridade de 53,3\%. Menores resultados foram obtidos por Polishook et al. (1996) estudando os folhedos de Guarea guidonea Sleumer e Manilkara bidentata Chev. obtiveram similaridade de fungos nas duas áreas estudadas $32,0 \%$ e $26,0 \%$, respectivamente.

A maioria das espécies encontradas neste estudo foi também registrada em trabalhos realizados em outras áreas de Mata Atlântica, (Barbosa et al. 2008; Gusmão et al. 2008; Cruz et al. 2007; Silva 2007; Grandi \& Silva 2006 e Grandi et al. 2002).

A distribuição dos táxons por classe de frequência evidenciou o predomínio de táxons esporádicos e pouco frequentes nas três espécies vegetais. Harleyodendron unifoliolatum apresentou táxons esporádicos $(82,0 \%)$ e pouco frequentes (18,0\%). Em Manilkara maxima 60,0\% dos táxons foram esporádicos e 30,0\% táxons foram pouco frequentes e $10,0 \%$ dos táxons foram frequentes (Beltraniella portoricensis, Gyrothrix magica, e Paraceratocladium polysetosum). A espécie Parinari alvimii apresentou $81,8 \%$ de táxons esporádicos e $18,2 \%$ de táxons pouco freqüentes (Tab. 3).

Analisando a distribuição dos táxons por classe de frequência nas áreas de estudo, observou-se como em relação às plantas, o predomínio de táxons esporádicos e pouco frequentes, apresentando somente um táxon frequente em cada área (Tab. 4). No PESC 78,0\% dos táxons foram esporádicos, $19,5 \%$ pouco frequentes e $2,5 \%$ frequente (correspondente a um táxon, Paraceratocladium polysetosum). No Ecoparque 70,3\% dos táxons foram esporádicos, 27,0\%

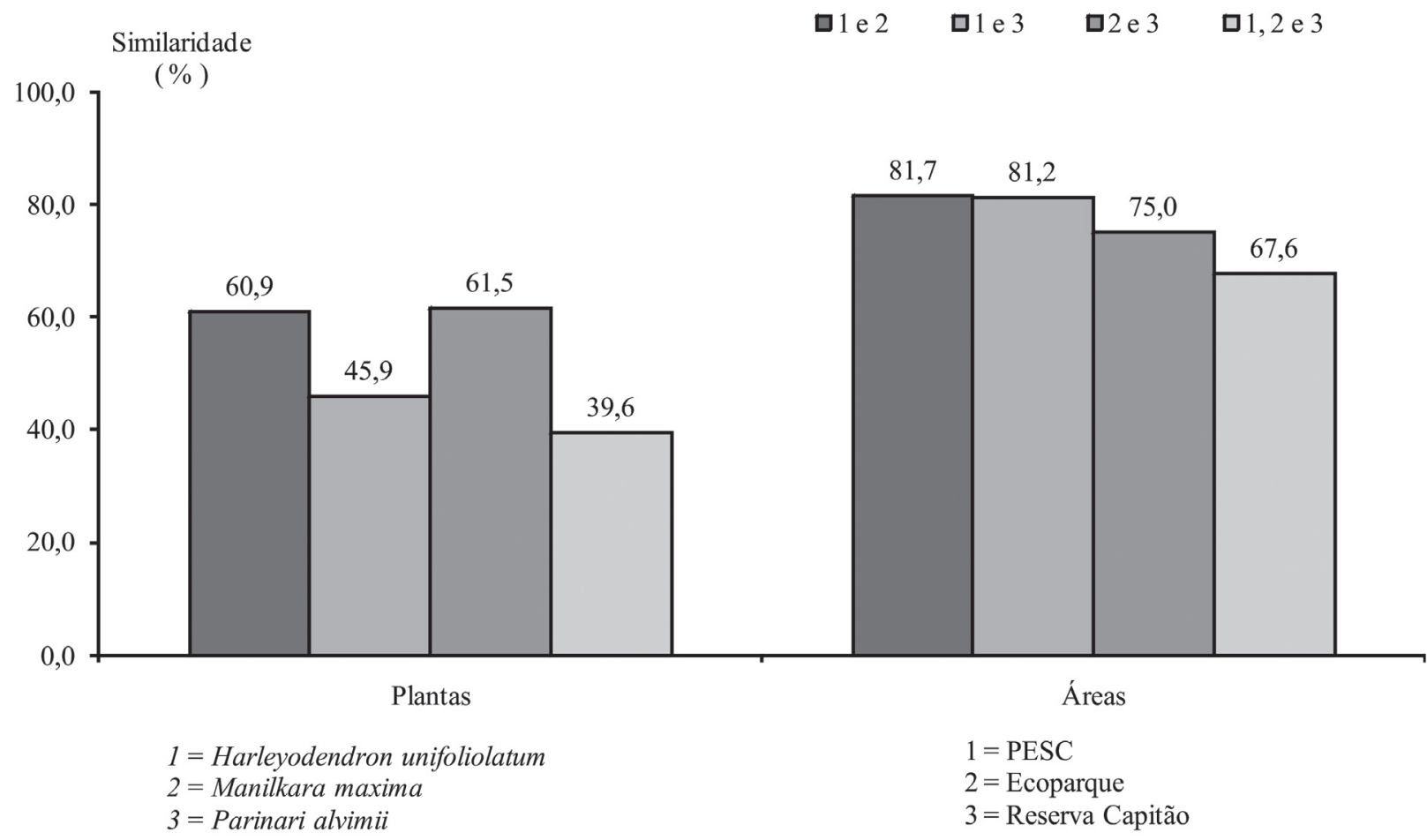

Figura 2. Similaridade fúngica encontrada entre as espécies vegetais Harleyodendron unifoliolatum Cowan, Manilkara maxima Penn. e Parinari alvimii Prance, e entre as áreas do PESC, Ecoparque e Reserva Capitão da Mata Atlântica do Sul da Bahia 
Tabela 3. Distribuição da frequência dos táxons, esporádica ( $\mathrm{F} \leq 10 \%)$, pouco frequente $(10<\mathrm{F} \leq 30 \%)$, frequente $(30<\mathrm{F} \leq 70 \%)$ e muito frequente ( $\mathrm{F}>70 \%)$, obtidos na serrapilheira de Harleyodendron unifoliolatum Cowan, Manilkara maxima Penn. e Parinari alvimii Prance

\begin{tabular}{|c|c|c|c|c|c|c|}
\hline Freqüência & $\begin{array}{c}\text { Harleyodendron } \\
\text { unifoliolatum }\end{array}$ & $(\%)$ & Manilkara maxima & $(\%)$ & Parinari alvimii & (\%) \\
\hline Esporádica & 32 & 82,0 & 18 & 60,0 & 18 & 81,8 \\
\hline Pouco frequente & 7 & 18,0 & 9 & 30,0 & 4 & 18,2 \\
\hline Frequente & 0 & 0 & 3 & 10,0 & 0 & 0 \\
\hline Muito frequente & 0 & 0 & 0 & 0 & 0 & 0 \\
\hline Riqueza / planta & 39 & 100 & 30 & 100 & 22 & 100 \\
\hline
\end{tabular}

Tabela 4. Distribuição da frequência dos táxons, esporádica $(\mathrm{F} \leq 10 \%)$, pouco frequente $(10<\mathrm{F} \leq 30 \%)$, frequente $(30<\mathrm{F} \leq 70 \%)$ e muito frequente $(\mathrm{F}>70 \%)$, obtidos nas áreas da Mata Atlântica do Sul da Bahia PESC, Ecoparque e Reserva Capitão

\begin{tabular}{lcccccc}
\hline Freqüência $^{*}$ & PESC & $(\%)$ & Ecoparque & (\%) & R. Capitão & $(\%)$ \\
\hline Esporádica & 32 & 78,0 & 26 & 70,3 & 28 & 87,5 \\
Pouco frequente & 8 & 19,5 & 10 & 27,0 & 3 & 9,4 \\
Frequente & 1 & 2,5 & 1 & 2,7 & 1 & 3,1 \\
Muito frequente & 0 & 0 & 0 & 0 & 0 & 0 \\
Riqueza / área & 41 & 100 & 37 & 100 & 32 & 100 \\
\hline
\end{tabular}

\section{Harleyodendron unifoliolatum}

Constância

$(\%)$

Acidental

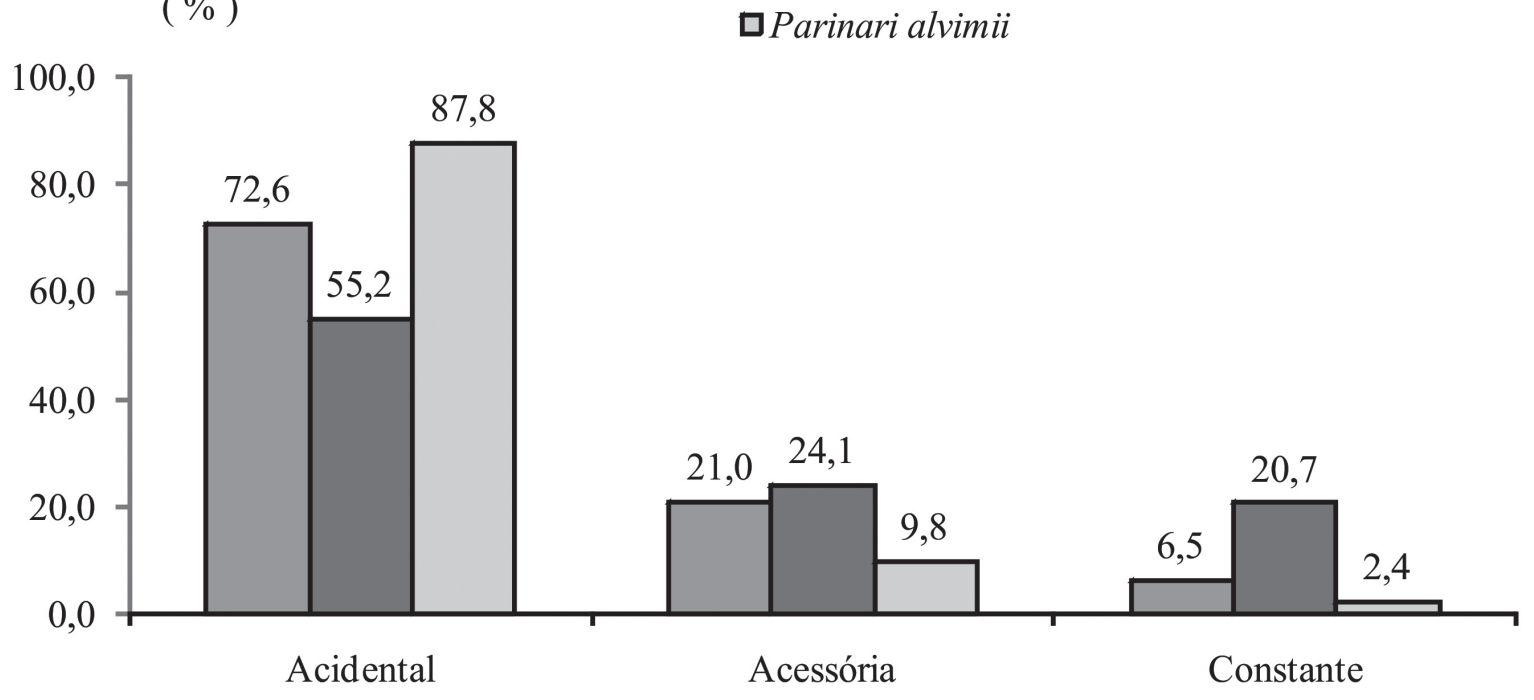

$\square$ Manilkara maxima

$\square$ Parinari alvimii

Constância entre as plantas

Figura 3. Constância dos fungos identificados na serapilheira de Harleyodendron unifoliolatum Cowan, Manilkara maxima Penn. e Parinari alvimii Prance

pouco frequentes e apenas um táxon (Gyrothrix magica) foi frequente (2,7\%). A Reserva Capitão apresentou $87,5 \%$ de táxons esporádicos, $9,4 \%$ pouco frequentes e $3,1 \%$ frequente (Volutella sp.).

Tanto na análise da frequência fúngica nas plantas, quanto nas áreas de estudo predominaram táxons esporádicos, seguido de táxons pouco frequentes, estes resultados corroboram com os de Barbosa (2007) que obteve predomínio de táxons esporádicos e pouco frequentes (13 táxons frequentes para C. melchiori e 9 táxons para C. nemorosa) e por Bills \& Polishook (1994) na Costa Rica que obtiveram seis táxons frequentes dos 178 táxons encontrados em folhedos.

Em Harleyodendron unifoliolatum 72,6\% dos táxons foram acidentais e 6,5\% constantes; em Manilkara maxima obteve-se 55,2\% de táxons com constância acidental e 20,7\% de táxons constantes e em Parinari alvimii 87,8\% dos táxons foram acidentais e 2,4\% constantes (Fig. 3). Nas áreas de estudo também predominaram os táxons acidentais. No 
PESC, $65,6 \%$ dos táxons foram acidentais e 6,3\% constantes; no Ecoparque 70,7\% dos táxons foram acidentais e 13,8\% de táxons constantes; na Reserva Capitão 78,0\% dos táxons foram acidentais e 12,2\% constantes (Fig. 4). Quanto à constância foram observados poucos táxons constantes como Beltrania rhombica, Cryptophiale kakombensis, Cryptophiale udagawae, Gyrothrix mágica, Inesiosporium longispirale e Volutella sp..

Em síntese a análise conjunta dos dados demonstra que a serapilheira das plantas nativas da Mata Atlântica do Sul da Bahia, Harleyodendron unifoliolatum, Manilkara maxima e Parinari alvimii abriga uma riqueza expressiva de fungos decompositores, diferenciada por espécie vegetal e pelas características estruturais das folhas de cada uma. Principalmente de Parinari alvimii que deve necessitar de maior tempo para a colonização dos fungos decompositores. A maioria dos táxons apresenta constância esporádica e frequência acidental. As Unidades de conservação da Mata Atlântica estudadas apresentaram uma riqueza semelhante em termos percentuais e similaridade fúngica. Este estudo sendo pioneiro na Mata Atlântica do Sul da Bahia e nas plantas Harleyodendron unifoliolatum, Manilkara maxima e Parinari alvimii, acima de tudo, vem mostrar a importância de se conhecer cada vez mais os fungos existentes neste Bioma, riquíssimo em biodiversidade, pouco conhecido cientificamente e constantemente ameaçado de extinção pelas atividades antrópicas. Desta forma importantes espécies podem ser perdidas com conseqüências desconhecidas para a manutenção da vida nos ecossistemas.

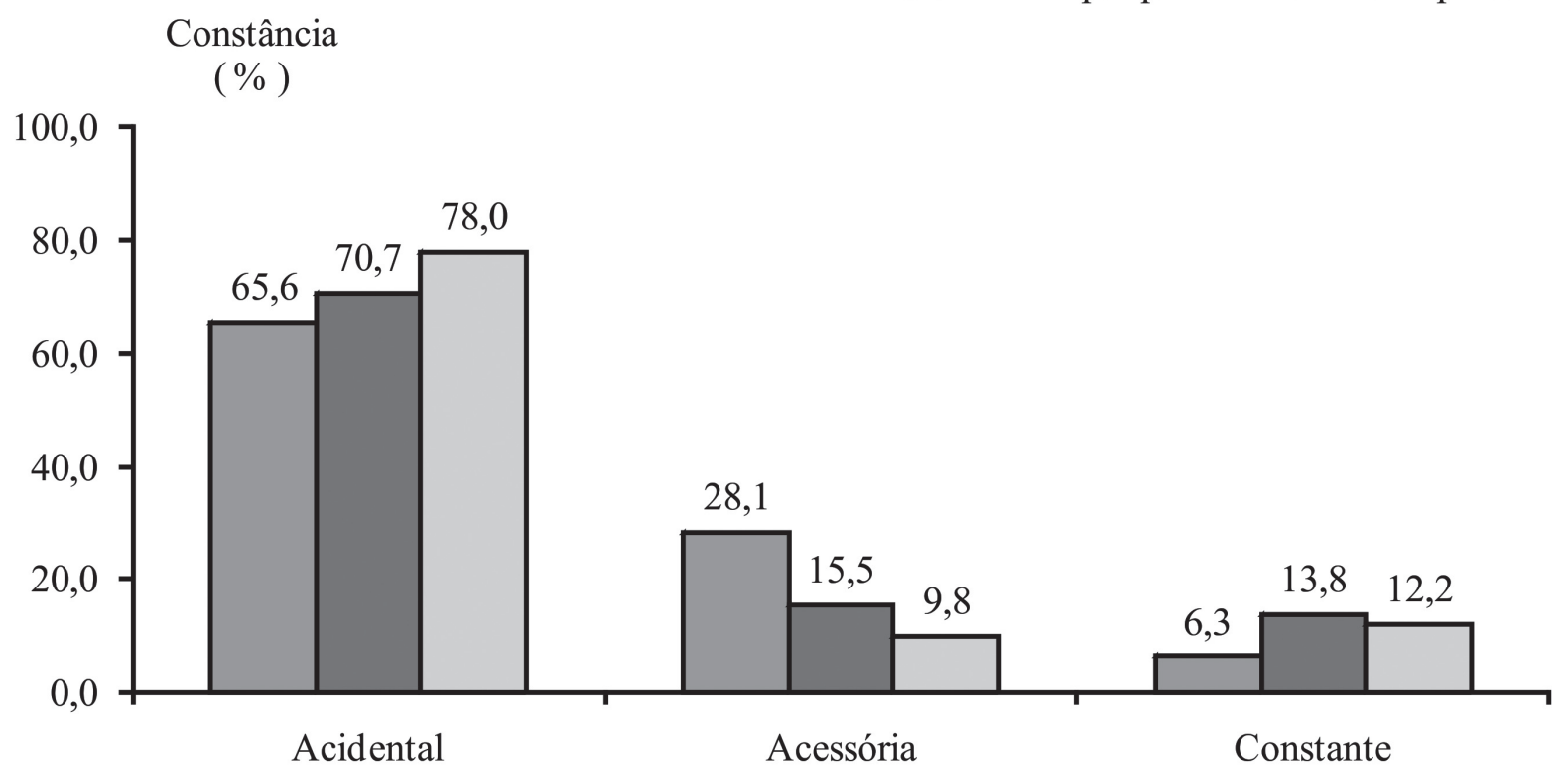

Constância entre as áreas

Figura 4. Constância dos fungos identificados nas áreas: PESC, Ecoparque e Reserva Capitão da Mata Atlântica do Sul da Bahia

\section{Agradecimentos}

O primeiro autor agradece a Comissão Executiva do Plano da Lavoura Cacaueira pela utilização dos laboratórios do Centro de Pesquisa para a realização deste trabalho, ao Instituto de Estudos Socioambientais do Sul da Bahia (IESB) e a Secretaria Estadual do Meio Ambiente da Bahia por autorizarem o estudo nas áreas de preservação ambiental. A Flávia Rodrigues Barbosa pelo auxílio na identificação dos fungos e envio de artigos, e José Lima Paixão pela identificação das plantas nas coletas.

\section{Referências bibliográficas}

Araujo, M.; Rocha, R.; Alger, K. \& Mesquita, C.A.B. , 1998. A Mata Atlântica Sula da Bahia: Situação atual, ações e perspectivas. Séries
Estado e Regiões da RBMA, Reserva da Biosfera da Mata Atlântica. São Paulo, MAB/UNESCO.

Barbosa, F.R. 2007. Fungos conidiais associados a folhas em decomposição de Clusia melchiori Gleason e C. nemorosa G. Mey em fragmento de Mata Atlântica, Bahia, Brasil. Dissertação de Mestrado em Biologia de Fungos. Recife, Universidade Federal de Pernambuco.

Barbosa, F.R.; Gusmão, L.F.P. \& Barbosa, F.F. 2008. Fungos anamórficos (Hyphomycetes) no Semi-árido do Estado da Bahia, Brasil. Acta Botanica Brasilica. 22(1): 29-36.

Barbosa, F.R.; Maia, L.C. \& Gusmão, L.F.P. 2009. Fungos conidiais associados ao folhedo de Clusia melchiorii Gleason e C. nemorosa G. Mey. (Clusiaceae) em fragmento de Mata Atlântica, BA, Brasil. Acta Botânica. Brasilica. 23(1): 79-84.

Bergamin, A.F. \& kimati, H. 1995. Variedades resistentes. Pp.297-324. In: Galli, F. (Ed.) Manual de fitopatologia: princípios e conceitos. 3. ed. São Paulo, Ceres.

Bills, G.F. \& Polishook, J.D. 1994. Abundance and diversity of microfungi in leaf litter of a lowland rainforest in Costa Rica. Mycologia. 86: 187-198.

Calduch, M.; Gene, J.; Stchigel, A.M. \& Guarro, J. 2002. New species of 
Dictyochaetopsis and Paraceratocladium from Brazil. Mycologia 94(6): 1071-1077.

Castañeda-Ruiz, R.F.; Gene, J. \& Guarro, J. 2001. A new species of Rhexoampullifera from leaf from Brazil. Mycologia 93(1): 168-170.

Castañeda-Ruiz, R.F.; Guarro, J.; Velázquesz-Noa, S. \& Gene, J. 2003. A new species of Minimelanolocus and some Hyphomycetes from rain Forest in Brazil. Mycotaxon 85: 231-239.

Castañeda-Ruiz, R.F.; Gusmão, L.F.P.; Heredia-Abarca, G. \& Saikawa, M. 2006. Some Hyphomycetes from Brazil. Two new species of Brachydesmiella. Two new combinations for Repetophragma, and new records. Mycotaxon 95: 261-270.

Cavalcanti, L.H. \& Mobin, M. 2004. Myxomycetes associated with palm trees at the Sete Cidades National Park, Piauí State, Brazil. Systematics and Geography of Plants 74: 109-127.

César, O. 1993. Produção de serapilheira na mata mesófila semidecídua da fazenda Barreiro Rico, município de Anhembi (SP). Revista Brasileira de Biologia 53(4), p.671-681.

Conservation International do Brasil, Fundação SOS Mata Atlãntica, Fundação Biodiversitas, Instituto de Pesquisas Ecológicas, Secretaria do Meio Ambiente do Estado de São Paulo, Semad/Instituto Estadual de Florestas - MG. 2000. Avaliação e Ações Prioritárias para a Conservação da Biodiversidade da Mata Atlântica e Campos Sudesteinos. Brasília, MMA/SBF.

Conservation International do Brasil, 2003. Disponível em: http://www. conservation.org.br/onde/mata_atlantica. Acesso em 03/06/2007.

Conti, J.B. \& Furlan, S.A. 2003. Geoecologia: o clima, os solos e a biota. Pp. 67-237. In: Ross, J.L.S. (Org.). Geografia do Brasil. São Paulo, EDUSP.

Cruz, A.C.R. da.; Marques, F.O. \& Gusmão, L.F.P. 2007. Fungos anamórficos (Hyphomycetes) da Chapada Diamantina: novos registros para o Estado da Bahia e Brasil Acta Botanica Brasilica 21(4): 847-855.

Dajoz, R. 1983. Ecologia Geral. 4. ed. Rio de Janeiro, Vozes.

Dix, N.I. \& Webster, J. 1995. Fungal Ecology. Cambridge, Cambridge University Press.

Gouvêa, J.B.S.; Silva, L.A.M.; Hori, M. 1976. Fitogeografia. In: Diagnóstico Socioeconômico da Região Cacaueira. CEPLAC/IICA. Recursos florestais 7: 1-7.

Grandi, R.A.P. \& Gusmão, L.F.P. 2002. Hyphomycetes decompositores do folhedo de Tibouchina pulchra Cogn. Revista Brasileira de Botânica 25: 79-87.

Grandi, R.A.P. \& Silva, T.V. 2006. Fungos Anamorfos decompositores do folhedo de Caesalpinia chinata Lam. Revista Brasileira de Botânica 29: 275-287.

Gusmão, L.F.P.; Góes Neto, A. \& Cruz, A.C.R. 2005. Fungos. Pp.227-236. In: Juncá, A.F., Funch, L. \& Rocha, W. (Orgs.). Biodiversidade e Conservação da Chapada Diamantina. Brasília, Ministério do Meio Ambiente.

Gusmão, L.F.P.; Ferreira, S.M.L.; Marques, M.F.O. \& Almeida, D.A.C. de. 2008. New species and records of Paliphora from the Brazilian semiarid region Mycologia 100(2): 306-309.
Maia, L.C. 1983. Sucessão de fungos em folhedo de Floresta Tropical Úmida. Recife, Ed. Universitária.

Maia, L.C. 2003. Coleções de fungos nos herbários brasileiros: estudo preliminar. Pp. 21-40. In: Peixoto, A.L. (Org.). Coleções Biológicas de apoio ao inventário, uso sustentável e conservação da Biodiversidade. Rio de Janeiro, Instituto de Pesquisa Jardim Botânico do Rio de Janeiro.

Mandarino, E.P. 1981. Implantação de cacaueiros sob mata raleada nas condições da Bahia. Boletim Técnico n. 85. Ilhéus, CEPLAC.

Marques, M.F.O.; Gusmão, L.F.P. \& Maia, L.C. 2008. Riqueza de espécies de fungos conidiais em duas áreas da Mata Atlântica no Morro da Pioneira, serra da Jibóia, BA, Brasil. Acta Botanica Brasilica 22(4): 954-961.

Martini, A.M.Z.; Fiaschi, P.; Amorim, A.M. \& Paixão, J.L. 2007. A hotpoint within a hot-spot: a high diversity site in Brazil's Atlantic Forest. Biodiversity and Conservation 16: 3111-3128.

Milanez, A.I.A. 1995. Atuação dos fungos nos ecossistemas. Pp. 67-72. In: Congresso da Sociedade Botânica de São Paulo, 9. Anais..., São Paulo, SBSP, FAPESP.

Muller-Dombois, D. 1981. Ecological measurements and microbial populations. Pp.173-184. In: Wicklow, D.T. \& Carroll, G.C. (Eds.). The fungal community: Its organization and role in the ecosystem. New York, Marcel Derker.

Myers, N. 1988. Threatened biotas: "hot-spots" in tropical rain forests. Environmentalist 8: 187-208.

Myers, N. 1990. The biodiversity challenge: expanded hot-spots analysis. Environmentalist 10: 243-56.

Polishook, J.D.; Bills, G.F. \& Lodge, D.J. 1996. Microfungi from decaying leaves of two rain forest trees in Puerto Rico. Journal of Industrial Microbiology 17: 284-294.

Queiroz, A.F. 1999 Dinâmica da ciclagem de nutrientes contidos na serapilheira em um fragmento de mata ciliar no Estado de São Paulo. Dissertação de Mestrado em Agronomia. Botucatu, Universidade Estadual de São Paulo.

Scott, J.M. Csuti, B.; Jacobi, J.D. \& Estes, J.E. 1987. Species richness. A geographic approach to protecting future biological diversity. BioScience 37: 782-88.

Silva, P. da. 2007. Fungos anamorfos decompositores do folhedo de Caesalpinia echinata Lam. Provenientes de exemplares estabelecidos em áreas com e sem impacto de poluição aérea. (Dissertação Mestrado em Biodiversidade Vegetal e Meio Ambiente). São Paulo, Instituto de Botânica da Secretaria do Meio Ambiente.

Silva, L. F. \& Mendonça, J. R. 2000. Terras avistadas por Cabral (Mata Atlântica 500 anos de devastação. Ilhéus, Ed. Editus.

Symonides, E. 1992. Biological diversity: The role of its evaluation and conservation in the Polish national parks. Wiadomosci Ekologiczne 38: 221-237.

Trappe, J.M. \& Schenck, N.C. 1982. Taxonomy of the fungi forming Endomycorrhizae. In: Schenck, N.C. (Ed). Methods and principles of Mycorrhizae research. The American Phytopatology. 